\title{
LES INTRODUCTIONS DE CRUSTACÉS DÉCAPODES D'EAU DOUCE EN FRANCE. PEUT-ON PARLER DE GESTION ?
}

\author{
E. VIGNEUX \\ Conseil Supérieur de la Pêche, Service de l'Informatique et des Relations Scientifiques, \\ Centre du Paraclet, B.P. 5, 80440 Boves, France.
}

\section{RÉSUMÉ}

La proportion d'espèces allochtones dans les peuplements français de crustacés décapodes d'eau douce est importante. Pour deux espèces de crevettes (famille des Atyidae) et trois espèces d'écrevisses (famille des Astacidae) autochtones, on compte quatre espèces d'écrevisses (famille des Astacidae et Cambaridae) et deux espèces de crabes importées (famille des Grapsidae et Potamonidae). S'appuyant sur des données d'inventaires nationaux, cette présentation dresse un rapide bilan du contexte écologique, réglementaire et humain.

Mots-clés : crustacés, décapodes, écrevisses, introduction, impacts, France.

\section{INTRODUCTIONS OF FRESHWATER DECAPOD CRUSTACEANS INTO FRANCE. CAN WE SPEAK OF MANAGEMENT ?}

\section{SUMMARY}

The proportion of foreign species in French populations of freshwater decapod crustaceans is important. For two native shrimp species (Atyidae family) and three native crayfish species (Astacidae family), we have four imported crayfish species (Astacidae and Cambaridae families) and two imported crab species (Grapsidae and Potamonidae families). Based on data from national inventories, this presentation draws up a short evaluation of the ecological, legislative and human background.

Key-words : crustaceans, decapods, crayfish, introduction, impacts, France.

\section{INTRODUCTION}

Malgré l'engouement suscité par les écrevisses et la curiosité ou l'étonnement inspirés par les crabes et les crevettes d'eau douce, les documents de synthèse faisant le point sur l'état des populations en France sont rares. Après plusieurs enquêtes lancées par l'administration des Eaux et Forêts (1959/1960) puis par le Ministère de l'Environnement avec l'aide du Conseil Supérieur de la Pêche (1977/1979 et 1988/1989), un véritable réseau d'observateurs s'est peu à peu structuré (NOËL, in LHONORE et al., 1993). A ce jour, les données de pêches de contrôle du Conseil Supérieur de la Pêche, les observations des gardes-pêche, les déclarations de scientifiques et de membres d'associations de protection de la nature sont stockées dans une banque de données (" Fauna-Flora ") gérée par le service du patrimoine naturel au Muséum National d'Histoire Naturelle (MNHN). Plus de 3000 stations et 16200 déclarations de présence sont ainsi référencées sur les crustacés décapodes en France. 
Un rapide bilan de la situation en France sera fait en s'appuyant sur cette base d'informations complétée par les données récentes d'une enquête " express " lancée en 1995 (CSP, 1996) sur les écrevisses (enquête effectuée par le service technique du Conseil Supérieur de la Pêche auprès de l'ensemble des brigades de gardes-pêche). Le contexte historique national et international ayant présidé aux introductions d'écrevisses est par ailleurs développé par LAURENT (1997).

\section{MÉTHODOLOGIE}

L'ensemble des données gérées dans la banque " Fauna-Flora " précédemment citée est traité en cartographie automatique par le MNHN. Chaque point figurant sur les cartes traduit l'existence d'au moins une observation dans un réseau de mailles de $0,2 \times 0,2$ grades découpant l'ensemble du territoire. En complément de ces informations, les départements où la présence d'écrevisses a été signalée dans l'enquête express 1995 sont identifiés sur les cartes. Ces informations, moins précises que la représentation par points, donnent cependant une indication précieuse sur l'évolution en cours.

Les opérations d'inventaires répétées dans le temps, permettant d'obtenir des séries chronologiques fiables afin de suivre la dynamique des populations en place, sont lourdes et coûteuses. Le gestionnaire est donc contraint à l'analyse d'enquêtes et de déclarations spontanées. L'hétérogénéité des sources d'informations rend l'exercice difficile. Dans ce contexte, le facteur humain peut induire un biais non négligeable : de protection ;

- réactions affectives entraînant la non-déclaration de populations autochtones par souci

- volonté de vérifier des hypothèses personnelles avec des espèces exotiques pas toujours avouables ;

- méconnaissance des critères de détermination.

Il est donc difficile de conclure à la disparition d'une espèce en s'appuyant sur la nondéclaration dans une enquête. A l'inverse, la déclaration d'une observation ne traduit pas nécessairement le scénario catastrophe d'une invasion généralisée. Compte tenu du référentiel retenu (maille de 0,2 $\times 0,2$ grades) et de la non-pondération des résultats de l'enquête par le nombre de cours d'eau visités, les cartes de présence / absence proposées ci-après ne permettent pas de rendre compte de l'intensité des mécanismes de régression ou de colonisation affectant les populations astacicoles. En gardant à l'esprit la prudence qui doit accompagner l'analyse d'enquêtes et de déclarations spontanées, cette démarche reste cependant, compte tenu des moyens disponibles, la seule possibilité d'analyse accessible au niveau national.

\section{LES POPULATIONS DE CRUSTACÉS DÉCAPODES AUTOCHTONES}

Au siècle dernier, les populations de crustacés décapodes vivant en eau douce n'étaient pas encore confrontées aux introductions d'autres espèces en provenance d'autres continents et moins soumises à la dégradation de leurs biotopes. On peut attribuer à nos peuplements autochtones deux espèces de crevettes (famille des Atyidae) et trois espèces d'écrevisses (famille des Astacidae). Les peuplements d'écrevisses étaient beaucoup plus abondants que de nos jours, essentiellement constitués de Austropotamobius pallipes pallipes Lereboullet, 1858 et, pour une moindre part, de Astacus astacus Linnaeus, 1758 et de Austropotamobius torrentium Schrank, 1803 (ces deux dernières étant situées à la limite occidentale de leur aire de répartition).

A. torrentium, signalée pour la première fois en Alsace par LEREBOULLET (1858) sous le nom d'Astacus longicornis (en raison de ses antennes particulièrement longues), fut considérée comme disparue puisque non signalée dans toutes les enquêtes effectuées (LAURENT et 
SUSCILLON, 1962 ; VIGNEUX, 1980 ; ARRIGNON, 1990). Avec sa redécouverte récente dans le département de la Moselle (MACHINO, 1995), on réalise qu'elle n'a probablement jamais disparu. Une autre population plus importante était identifiée dès 1990 dans le même département mais non signalée par mesure de protection. Aux dires des anciens, cette population était connue depuis toujours (COLLAS, comm. pers.). Les risques de méprise avec Austropotamobius pallipes permettent de penser que d'autres populations d'Austropotamobius torrentium ont pu être oubliées dans des départements voisins. Les Astacus astacus, facilement identifiables, n'ont pas fait l'objet de telles méprises. Elles sont depuis longtemps implantées dans les départements du Nord-Est de la France.

Les crevettes d'eau douce sont beaucoup plus discrètes. Atyaephyra desmaresti Millet, 1831 (ou caridine), dont la sous-espèce desmaresti est autochtone dans les départements méditerranéens (Fig. 1D), est une petite crevette d'une trentaine de millimètres de long affectionnant les eaux calcaires et vivant à l'abri des pierres et dans la végétation des berges. Naturellement implantée dans les départements du pourtour méditerranéen, elle a profité des canaux creusés par l'homme pour gagner le Nord de l'Europe (NOËL, in LHONORE et al., 1993).

La seconde crevette identifiée est cavernicole, Troglocaris inermis Fage, 1937, et limitée aux eaux souterraines de la zone karstique des départements du Gard et de l'Hérault. Cette espèce est le seul décapode souterrain endémique de France et mérite à ce titre une attention particulière (JUBERTHIE et JUBERTHIE-JUPEAU, 1975 ; JUBERTHIE-JUPEAU, in VIGNEUX et al., 1993).

\section{LA “ GESTION " ET LES TRANSFERTS DE POPULATIONS AUTOCHTONES ET PROCHES}

Faisant suite aux épizooties touchant les peuplements d'écrevisses autochtones depuis la fin du siècle dernier (ANDRÉ, 1960), de nombreuses tentatives de repeuplements ont donné lieu à des transferts de populations. Ces pratiques ont conduit au brassage des populations d'écrevisses à pieds blancs françaises, avec l'introduction de sous-espèces italiennes ( $A$. pallipes italicus) et originaires de la péninsule lbérique (A. pallipes lusitanicus) (Fig. 1A). Dans ce contexte, le caractère autochtone prend un sens plus flou. La possibilité d'hybridation entre sous-espèces et la variabilité des critères morphologiques entre isolats géographiques rendent le diagnostic très délicat.

L'isolement de l'ADN mitochondrial chez Austropotamobius pallipes pallipes et l'analyse de sa variabilité ouvrent de nouvelles perspectives pour l'avenir (GRANDJEAN et SOUTYGROSSET, 1996), mais déjà l'adaptation aux crustacés de techniques encore plus fines (microsatellites) paraît indispensable. Dans ce contexte où les peuplements natifs coexistent avec des populations transférées de même espèce, l'approche génétique s'avère précieuse pour comprendre la situation et tenter d'engager une gestion patrimoniale (en évitant les transferts inopportuns de populations). A l'évidence, cette gestion passe avant tout par la préservation des biotopes ou leur restauration. Il est également urgent d'analyser les causes de disparitions insidieuses affectant des populations que l'on croyait à l'abri dans les derniers refuges comme par exemple les bassins versants entièrement forestiers (faut-il incriminer l'enrésinement, la création de plans d'eau, l'emploi de produits de traitement du bois sur les lieux de stockage ou de scierie ?). II convient de souligner que la régression constante des peuplements naturels de $A$. pallipes pallipes depuis une trentaine d'années a conduit I'International Union for the Conservation of Nature and Natural Resources (GROOMBRIDGE, 1994) à inscrire cette écrevisse sur la liste rouge des espèces menacées. L'intégration de l'espèce ( $A$. pallipes) dans l'annexe 2 de la directive 92/43/CEE élaborée par la communauté européenne (Journal officiel des communautés européennes, 22/07/1992, article L 206, p. 7-38) et relative à la " conservation des habitats naturels de la faune et de la flore sauvages " va dans le même sens. L'intitulé de cette annexe est "Espèces animales et végétales d'intérêt communautaire dont la conservation nécessite la désignation de zones spéciales de conservation ". Cette directive prévoyant des mesures conservatoires proportionnées à la 
gravité de la situation intègre par ailleurs, dans son annexe 5, l'ensemble de nos trois astacidés autochtones sous l'intitulé "Espèces animales et végétales d'intérêt communautaire dont le prélèvement dans la nature et l'exploitation sont susceptibles de faire l'objet de mesures de gestion ". Cette directive exprime de façon fort opportune la nécessité d'une gestion patrimoniale intégrant la protection des espèces menacées et de leurs biotopes. On observera cependant que la rédaction de l'intitulé de l'annexe 5 laisse transparaître dans sa forme actuelle une notion de gestion largement répandue et que malheureusement on pratique encore, c'està-dire une simple réponse à un état de crise face à la menace d'une pénurie. S'agissant de Austropotamobius torrentium, considérée comme disparue en France depuis le siècle dernier, l'opportunité de mesures de protection plus strictes (relevant de l'annexe 4) mériterait d'être examinée (Fig. 1B). Les Astacus astacus ont également fait l'objet de transplantations sur le territoire national en dehors de leur aire de répartition naturelle (Fig. 1C).

Tout en restant au sens de la loi des espèces autochtones, les écrevisses à pieds blancs et les écrevisses à pieds rouges ont fait l'objet de nombreux transferts artificiels. Dans ce contexte, il paraît plus adapté pour les gestionnaires de raisonner en termes de peuplements autochtones (naturellement en place) plutôt que d'espèces autochtones (souvent transférées). La carte figure 2 montre malheureusement combien cette subtilité devient dérisoire face à la menace que représente la multiplication des introductions d'espèces exotiques. A ce jour, seul le département de la Lozère ne serait habité que de peuplements astacicoles considérés comme autochtones et strictement constitués d'Austropotamobius pallipes pallipes (ce qui n'exclut pas la présence de peuplements de ce type dans d'autres départements).

\section{LES INTRODUCTIONS DE CRUSTACÉS EXOTIQUES ET LA RÉGLEMENTATION}

Aux populations autochtones précédemment décrites ( 3 espèces d'écrevisses et 2 espèces de crevettes) viennent s'ajouter 2 espèces de crabes et 4 espèces d'écrevisses exotiques.

Le crabe chinois, Eriocheir sinensis Edwards, 1854, fut introduit en Europe au début du siècle. Probablement transporté à l'état larvaire dans les eaux de ballast des navires, on le signala pour la première fois en Allemagne dans le fleuve Aller en 1912. Après avoir colonisé tous les grands estuaires de la mer du Nord, de la Baltique, des côtes atlantiques et de la Manche, il fut signalé dans le Midi de la France où it semble à ce jour avoir disparu. Après une phase d'extension, on assiste semble-t-il à une diminution des peuplements depuis une quarantaine d'années (Fig. 11).

\section{Figure 1}

Distribution des peuplements de crustacés décapodes dans les eaux douces françaises et internationales.

La présence de peuplements au niveau national, enregistrée dans la banque "Fauna-Flora " (MNHN), est signalée par un point rouge par maille de 0,2 $\times 0,2$ grades.

La présence de peuplements dans l'enquête 1996 est signalée par le marquage en jaune des départements concernés.

Figure 1

Distribution of decapod crustacean populations in French and international freshwaters.

The presence of populations at the national level, as registered in the "Fauna-Flora " (MNHN) data bank, is indicated by a red dot for a grid of $0.2 \times 0.2$ grades.

The presence of populations in the 1996 inquiry is indicated by a yellow label of the concerned departments. 


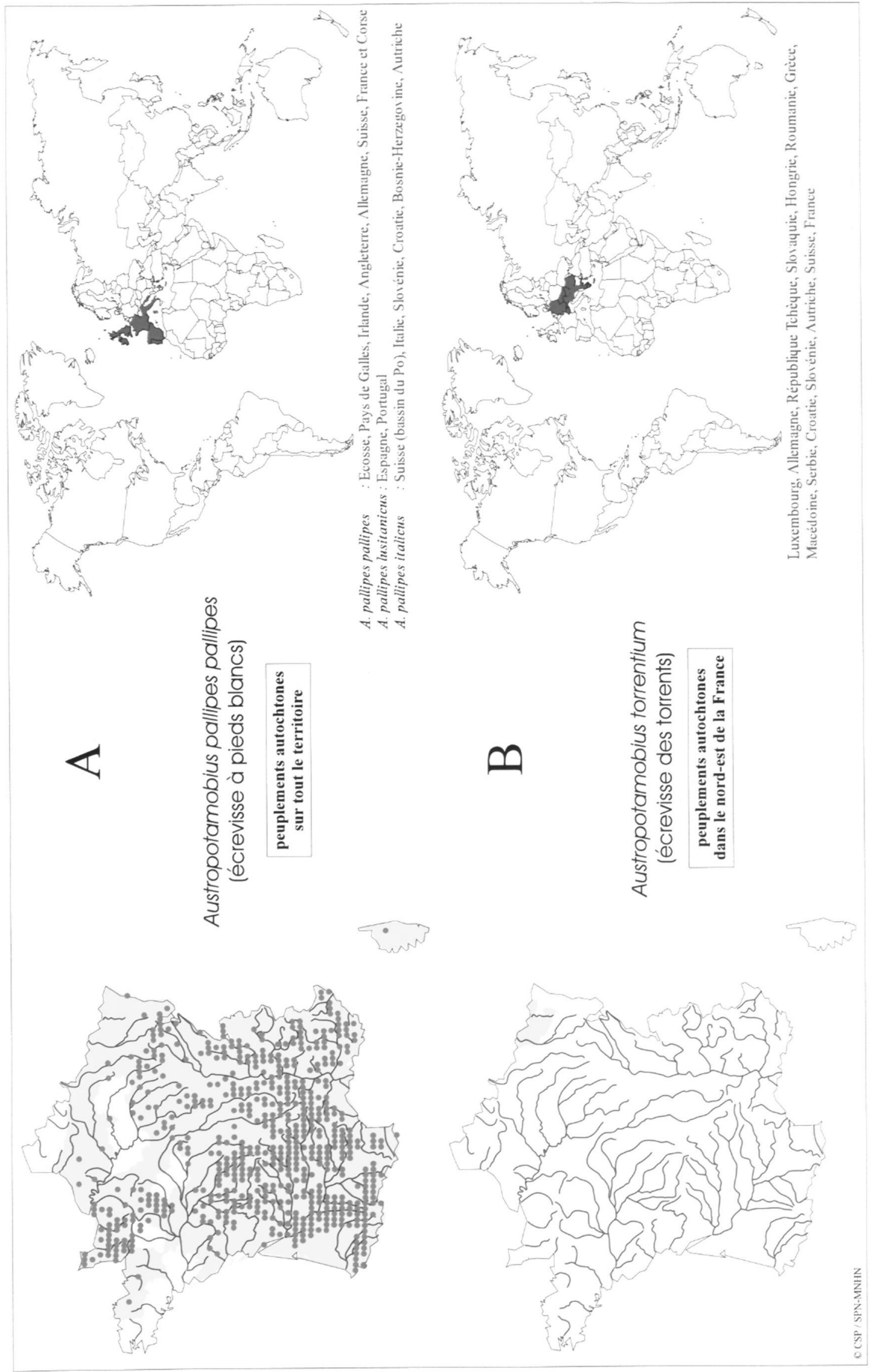




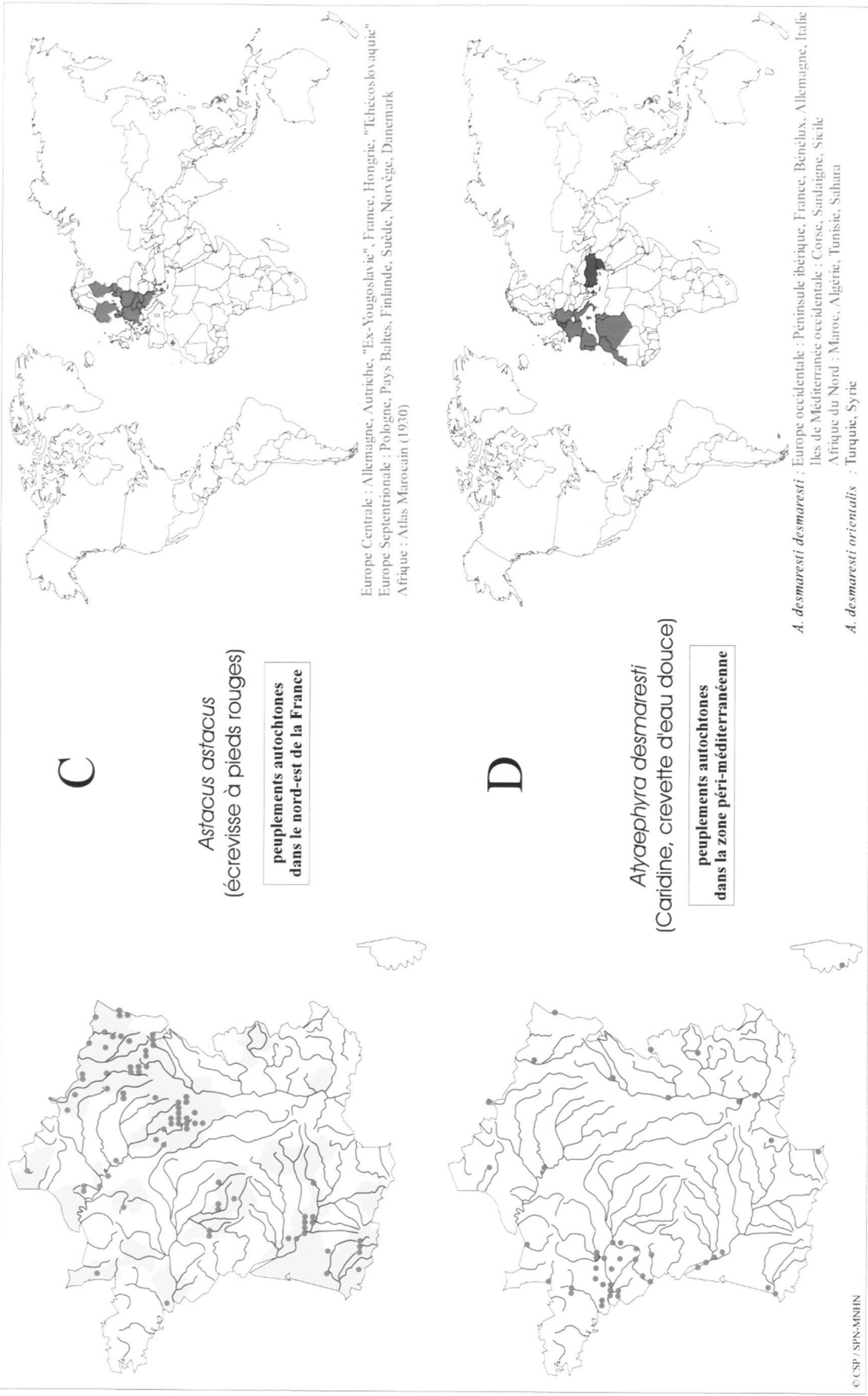




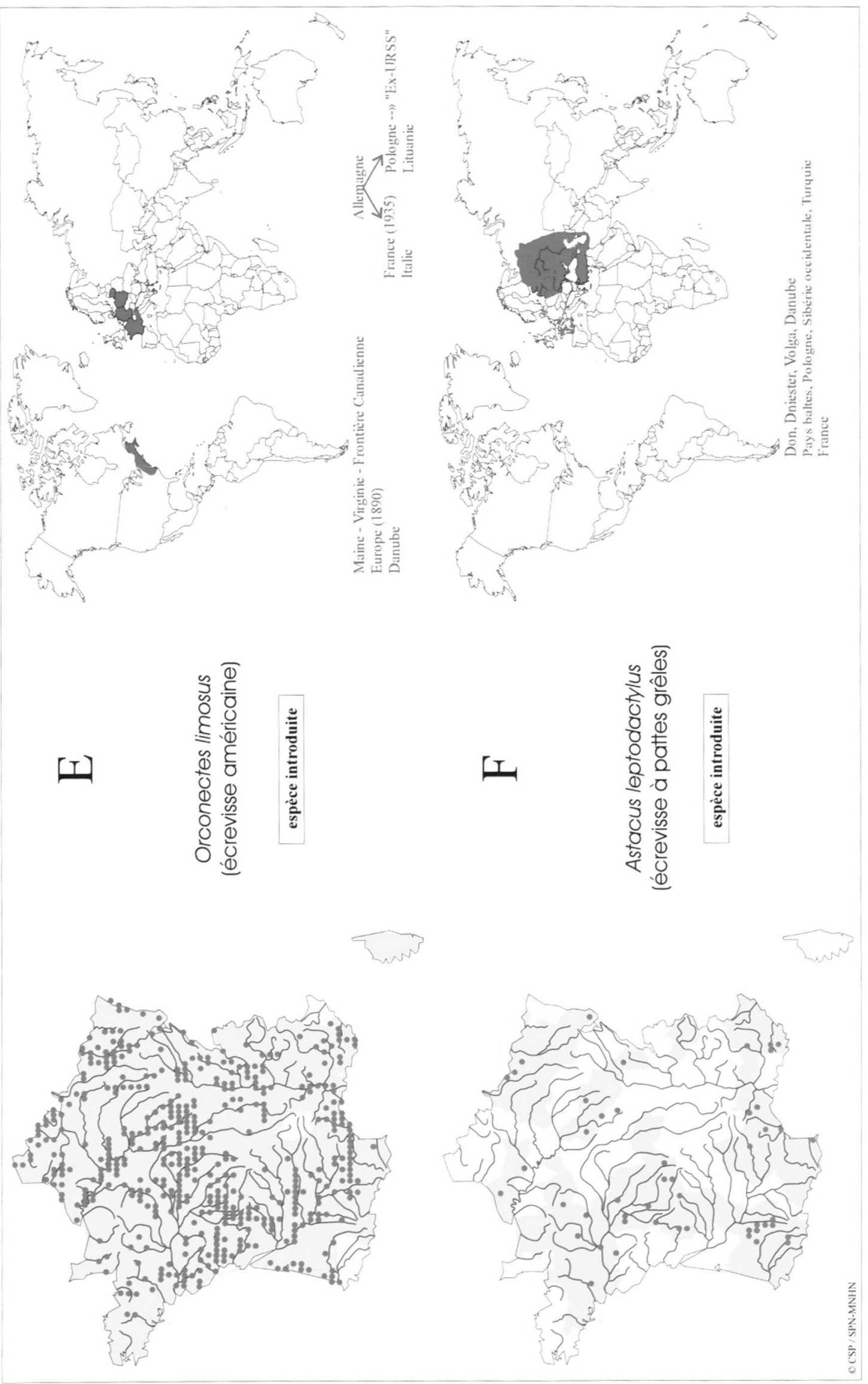




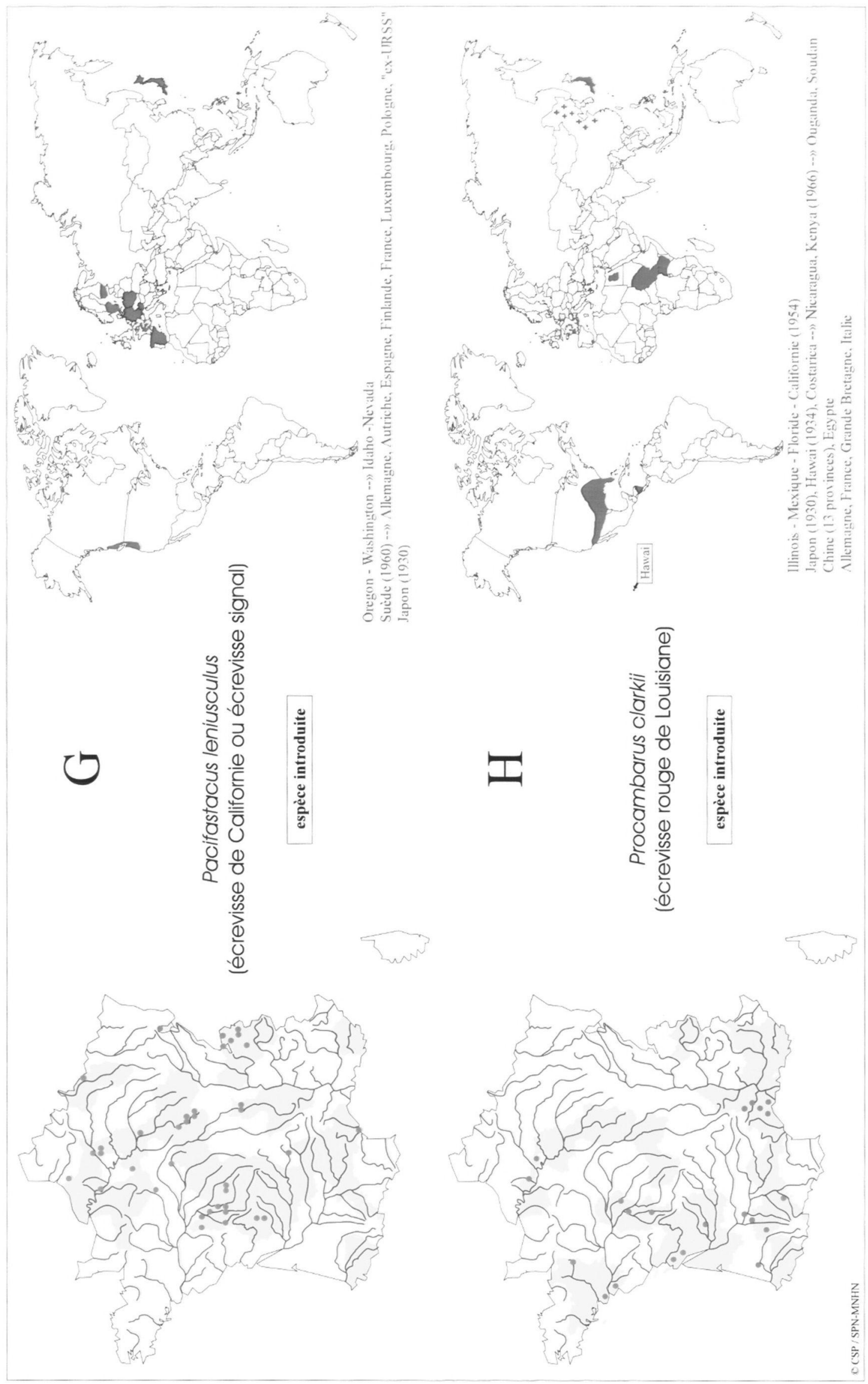




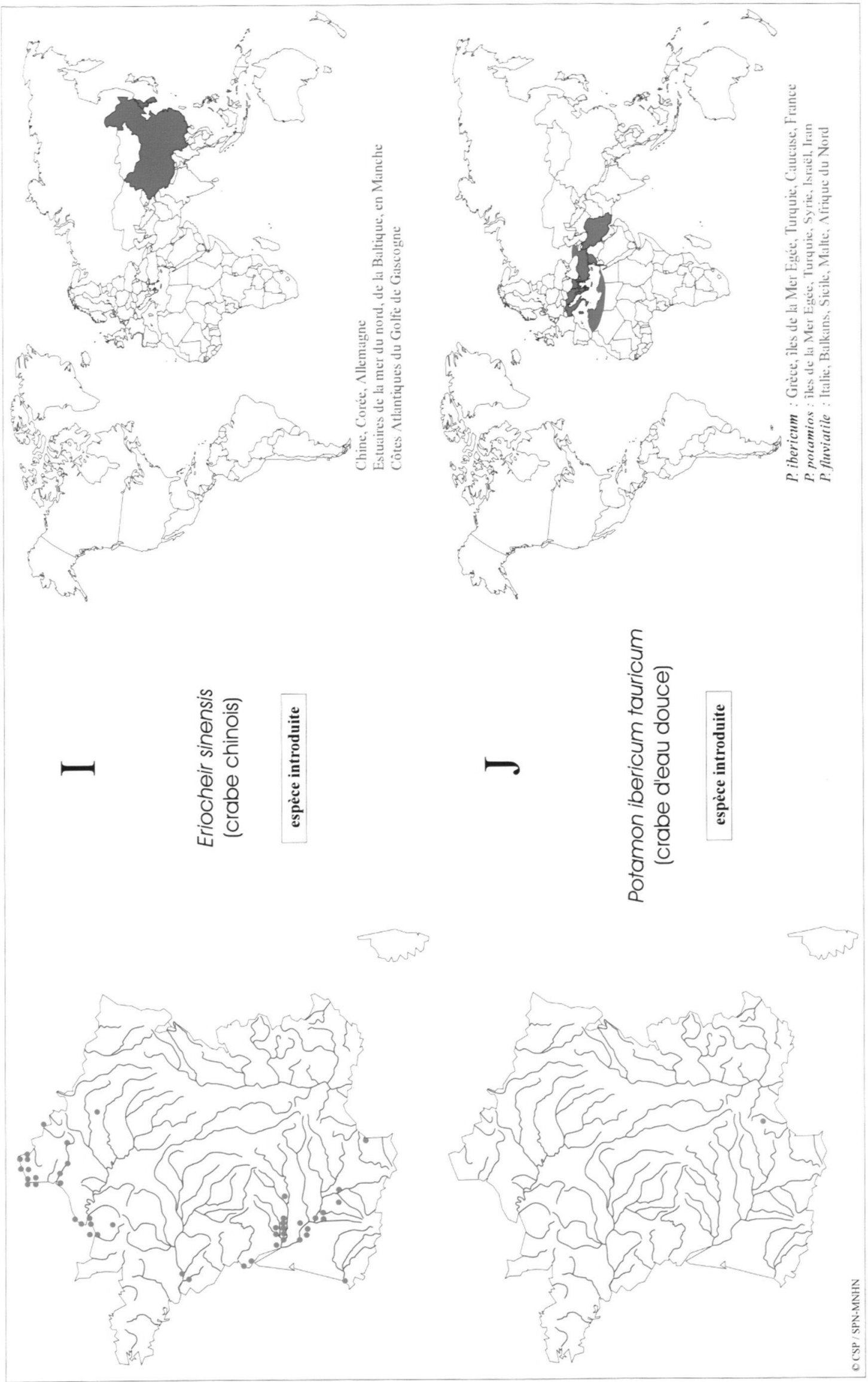




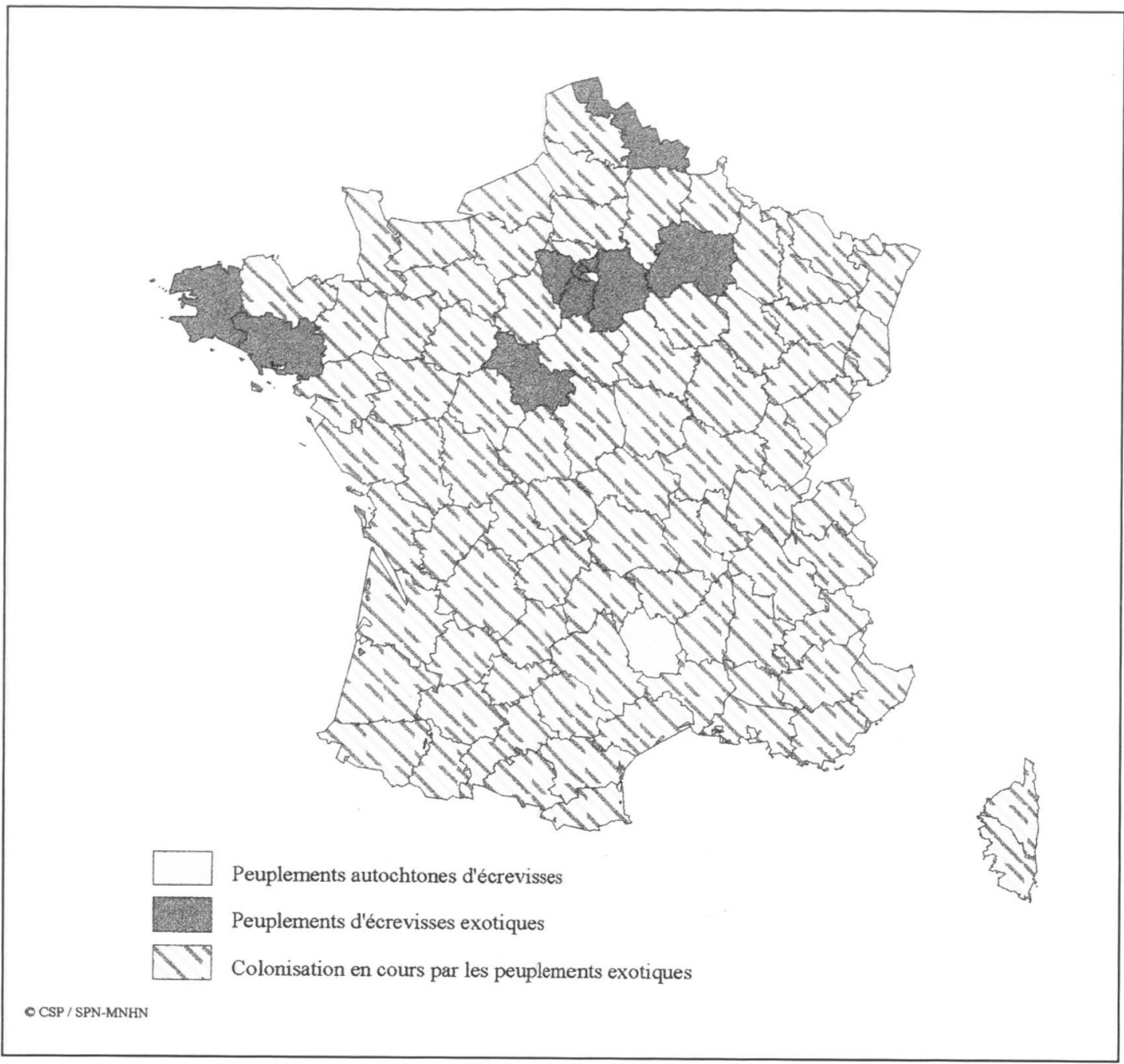

Figure 2

Distribution des peuplements d'écrevisses exotiques importées au sein des peuplements autochtones.

Figure 2

Distribution of imported exotic crayfish populations in native populations.

L'absence de prolifération du crabe chinois dans les zones colonisées par Orconectes limosus a inspiré l'hypothèse d'une compétition sévère (en faveur de cette écrevisse) pour le partage d'un même biotope (LAURENT et SUSCILLON, 1962). Par ailleurs, le crabe chinois, considéré comme un crustacé nuisible (creusant les berges et pouvant gêner le poisson et consommer les pontes), fut longtemps jugé comme inutilisable pour l'alimentation humaine. II s'est révélé ultérieurement être très prisé par les communautés asiatiques. Le contexte semble donc très favorable à la poursuite de sa raréfaction qui d'un point de vue écologique peut être considérée comme positive.

Le second crabe, originaire de Turquie, Potamon ibericum tauricum Czerniavsky, 1884, est beaucoup plus rare en France. Une seule population est recensée en France (CHARMANTIER, 1992). Cette espèce affectionne les eaux calmes ou stagnantes; en raison de sa faible représentation (Fig. $1 \mathrm{~J}$ ), elle peut être considérée plutôt comme une curiosité que comme un réel danger pour les populations autochtones. 
II n'en est malheureusement pas de même pour les peuplements d'écrevisses exotiques. Favorisées par une meilleure tolérance à la dégradation des milieux et par une meilleure résistance aux épizooties affectant nos populations autochtones, les écrevisses originaires du continent américain sont arrivées dans l'ordre suivant: Orconectes limosus Rafinesque, 1817, dès 1890 (Fig. 1E), sous le nom de Cambarus affinis (LEGER, 1924 ; BUFFAULT, 1925 ; ANDRÉ, 1960) ; Pacifastacus leniusculus Dana, 1852, en 1973 (Fig. 1G) ; Procambarus clarkii Girard, 1852, à partir de 1976 (Fig. 1H). En provenance de Turquie, Astacus leptodactylus fut également introduite dès la fin du 19ème siècle (Fig. 1F).

Soulignons l'effort de clarification du législateur qui, par le décret $n^{\circ}$ 94-978 du 10 novembre 1994 (JO du 13 novembre 1994), a corrigé certaines lacunes et simplifié notamment les mesures réglementant la capture des écrevisses. Quatre espèces profitent de mesures conservatoires dans ces nouvelles dispositions réglementaires, avec une période d'ouverture spécifique (Art. R 236-11) de 10 jours en juillet et une taille réglementaire (Art. R 236-23). Aux trois espèces autochtones s'ajoute ainsi Astacus leptodactylus assimilée comme telle par le législateur. Cette espèce inféodée aux eaux calmes et plutôt chaudes ne présente pas les inconvénients comportementaux des autres espèces importées (agressivité, creusement des berges, prolifération nuisible aux poissons) ni les résistances à certaines maladies (Aphanomycose) leur permettant de se comporter en porteurs sains, donc vecteurs potentiels d'épizooties. Par défaut, toutes les autres espèces sont considérées comme indésirables et à ce titre pêchables sans taille réglementaire toute l'année en seconde catégorie et du 11 mars au 17 septembre dans les rivières à truites (première catégorie) avec une interdiction de remise à l'eau et de transport vivant.

\section{DISCUSSION}

L'aire de répartition des organismes vivants n'est jamais figée, elle fluctue en fonction d'opportunités écologiques variées. Parfois la dynamique du système se trouve accélérée au hasard de la création de voies de communication (naturelles ou artificielles) privilégiant la colonisation. Ce fut le cas de crevettes d'eau douce (caridines) profitant de la création de voies navigables. D'autres facteurs peuvent intervenir pour inverser un processus de colonisation, la régression du crabe chinois après une phase d'extension semble être un exemple illustratif. S'agissant des peuplements astacicoles autochtones, l'accumulation de circonstances défavorables inspire un bilan préoccupant pour l'avenir. Aux phénomènes régulateurs naturels (sécheresse, épidémies) se sont ajoutés de nombreux problèmes inhérents aux activités humaines comme le révèlent les enquêtes:

- pollutions variées ;

- travaux de curage ;

- rectification de cours d'eau ;

- introduction du rat musqué ;

- repeuplements en truites.

Les études scientifiques manquent pour estimer objectivement l'importance relative de ces facteurs perturbants, mais l'examen des cartes composant la figure 1 permet à l'évidence d'ajouter un élément supplémentaire d'importance grandissante :

- la compétition avec les espèces exotiques (avec l'augmentation des risques pathologiques).

Ainsi, les phénomènes naturels de colonisation et de régulation des peuplements déjà perturbés par la dégradation des biotopes se trouvent redistribués selon de nouvelles règles échappant aux scientifiques, aux gestionnaires et probablement encore plus pénalisantes pour les espèces autochtones. Pendant des années, les scientifiques se sont appliqués à rechercher et à mettre en oeuvre des méthodes d'appréciation des caractéristiques biologiques des 
milieux à partir de l'analyse des communautés d'organismes vivants qu'ils hébergent. Les approches sont multiples et sortent du cadre de cet exposé (HUET, 1949 ; ILLIES et BOTOSANEANU, 1963 ; TUFFERY et VERNEAUX, 1967 ; VERNEAUX, 1973, 1976 ; PRYGIEL et COSTE, 1996 ; etc.). On considère à chaque fois que le peuplement étudié, qu'il soit composé de poissons, de macroinvertébrés, de diatomées ou autres, est le résultat d'une intégration complexe mais observable des interrelations avec le biotope et les autres communautés en place. Force est de constater que la multiplication des introductions de toutes natures d'espèces allochtones modifie en profondeur ce que l'on a coutume d'appeler des équilibres (à l'échelle de temps humaine) en compliquant sérieusement l'analyse de la répartition des taxons. Le déterminisme des changements affectant les communautés vivantes se trouve modifié par des interventions humaines multiples et peu prévisibles, ce qui complique d'autant la recherche de significations écologiques à la base des principes de gestion. Les populations astacicoles autochtones apparaissent dans ce contexte comme des indicateurs biologiques sous-étudiés, voire oubliés, en raison de la complexité des problèmes à appréhender et du peu de moyens disponibles pour ces travaux. La difficulté du problème posé par la préservation et la gestion du patrimoine astacicole ne doit pas pour autant inspirer la résignation mais plutôt la recherche de collaborations plus soutenues entre partenaires scientifiques, gestionnaires et collectivités régionales.

\section{CONCLUSION : ENJEUX, CONSÉQUENCES ET RECOMMANDATIONS}

On ne peut que constater le déséquilibre de cette présentation en faveur de la problématique astacicole. Celui-ci est en rapport avec l'intérêt suscité sur le terrain. A l'enjeu patrimonial s'ajoute l'attachement culturel ou sentimental de la pêche à l'écrevisse déchaînant parfois localement de véritables passions. On parle d'ailleurs le plus souvent de la pêche à l'écrevisse comme de la pêche à la truite, oubliant que derrière le mot écrevisse se cachent de nombreuses espèces aux comportements et aux facultés adaptatives très différents. On a souvent parlé de façon journalistique après l'introduction d'espèces exotiques du « retour de l'écrevisse " associé au cortège de symboles sur l'eau pure et les petits ruisseaux. Cette méconnaissance a encouragé les initiatives individuelles privilégiant l'introduction d'espèces exotiques moins polluosensibles au détriment de la restauration des populations autochtones et de la réhabilitation des cours d'eau astacicoles d'origine. Les mots-clés du séminaire sur les introductions d'espèces à l'origine de cette présentation sont " enjeux, conséquences, recommandations ". Avec la gestion des populations astacicoles, l'enjeu patrimonial se heurte à l'enjeu commercial. Les projets d'astaciculture sont nombreux et les objectifs touristiques, gastronomiques, écologiques suivent parfois des logiques antagonistes. Partant de la conséquence la plus évidente (la prolifération des espèces exotiques), il est difficile de passer sous silence les véritables causes. Au delà de la dégradation des milieux souvent perçue comme une fatalité, l'analyse, la plus succincte soit-elle, conduit inévitablement au principal acteur, le comportement humain. Sans vouloir réduire l'être humain à un système binaire, on constate que son comportement est souvent gouverné par deux grandes tendances : le jeu et la recherche du profit. Ces deux penchants peuvent curieusement déboucher sur des attitudes aussi bien protectionnistes que destructrices. L'attrait permanent du « nouveau ", la recherche de la "curiosité " comme l'écrevisse géante de Tasmanie (Astacopsis gouldi Clark) ou de l'animal à la croissance impressionnante facile à élever comme les Cherax d'Australie attisent ces deux penchants. Mais l'écrevisse autochtone peut devenir " nouvelle " dans l'esprit de celui qui l'avait oubliée, comme la gastronomie à l'ancienne revient périodiquement à la mode.

Ces deux penchants sont également influencés par deux autres caractéristiques : l'impatience et la recherche de facilité ou l'insouciance. II est plus facile d'introduire une espèce résistante à la pollution que de se débarrasser de la pollution. La gestion étant une pratique permettant de privilégier certains objectifs, il existe en réalité de nombreuses gestions. Celle que nous préconisons par humilité vis-à-vis des processus naturels dont nous ne sommes qu'un résultat est plutôt de type patrimonial, mais on pourrait défendre avec autant de pertinence la thèse inverse. Le dernier mot-clé est l'exercice le plus difficile de cette réflexion : 
" recommandations ". La notion de " recommandation " découle d'un choix de gestion. Le principe de gestion suppose quant à lui la maîtrise de la situation, ce qui est loin d'être le cas avec les introductions d'espèces. Dans notre logique, celle-ci doit néanmoins tout mettre en oeuvre pour éviter de nouvelles introductions sauvages. La réglementation va dans ce sens de même qu'il est illégal de polluer. Depuis la reconnaissance d'utilité publique bien compréhensible à l'époque (1855) de la Société Impériale zoologique d'acclimatation créé par Geoffroy Saint-Hilaire les temps ont changé. Dans une pétition circulant sur Internet plus de 400 naturalistes américains protestent contre les introductions d'espèces allochtones (auxquelles on attribuerait le déclin de $42 \%$ d'espèces menacées et la perte annuelle de plusieurs centaines de milliers de dollars) (TARDIEU, 1997). La relation de force qui parfois s'installe entre la gestion productiviste et la gestion patrimoniale nous semble construite sur un quiproquo, une divergence dans la notion de rentabilité opposant le court terme au long terme. Comment situer l'action des gestionnaires entre les techniques de pointe de biomanipulation (écologiques ou génétiques) des scientifiques et l'insouciance de l'expérimentateur en herbe qui importe des espèces exotiques pour voir ou pour essayer?

On ne peut qu'espérer que chacun prenne conscience de ses responsabilités individuelles, car ce n'est pas en recherchant des culpabilités que l'on trouve des solutions. Par des contacts soutenus entre scientifiques et gestionnaires en privilégiant la vulgarisation et l'information réciproque, on pourra créer les conditions de la prise de responsabilité de chacun.

\section{BIBLIOGRAPHIE}

ANDRÉ M., 1960. Les écrevisses françaises. Edition Lechevallier, 293 p.

ARRIGNON J., 1990. L'enquête nationale sur les écrevisses, une réglementation inadaptée. Eaux libres, 2.

BUFFAULT P., 1925. Une écrevisse américaine acclimatée en France : le cambarus du Cher. Rev. des Eaux et Forêts, 63, 274.

CHARMANTIER G., 1992. Occurrence of freshwater crabs, genus Potamon, in southern France. Journal of crustacean biology, 12 (4), 620-626.

CSP, 1996. Premiers résultats de l'enquête express écrevisses. Circulaire D003, Direction Générale, Paris, $5 \mathrm{p}$.

CUKERZIS J., 1984. La biologie de l'écrevisse (Astacus astacus L.). INRA Publications, $313 \mathrm{p}$.

GRANDJEAN F., SOUTY-GROSSET C., 1996. Isolation and characterization of mitochondrial DNA from the endangered white-clawed crayfish Austropotamobius pallipes pallipes Lereboullet, 1858. Bull. Fr. Pêche Piscic., 343, 175-182.

HUET M., 1949. Aperçu des relations entre la pente et les populations piscicoles dans les eaux courantes. Trav. Stat. Rech. Goenendaal, 4 (13), 232-243.

ILLIES J., BOTOSANEANU L., 1963. Problèmes et méthodes de la classification et de la zonation écologique des eaux courantes considérées surtout du point de vue faunistique. Mitt. internat. Verein. Limnol., 12, 1-57.

GROOMBRIDGE, 1994. International Union for the Conservation of Nature, Red list of threatened animals. Groombridge B. ed., Switzerland and Cambridge, United Kingdom, 286 p.

JUBERTHIE C., JUBERTHIE-JUPEAU L., 1975. La réserve biologique du laboratoire souterrain du CNRS à Sauve (Gard). Annales de Spéléologie, 30 (3), 539-551.

LAURENT P.J., 1997. Introductions d'écrevisses en France et dans le monde, historique et conséquences. Bull. Fr. Pêche Piscic., 344-345.

LAURENT P.J., SUSCILLON M., 1962. Les écrevisses en France. Ann. St. Cent. d'Hydrobiol. Appliq., 9, 335-395. 
LEGER L., 1924. Une nouvelle écrevisse dans les eaux françaises. C. R. Acad. Sciences, 179, 1205.

LEREBOULLET A., 1858. Description de deux nouvelles espèces d'écrevisses de nos rivières. Mem. Soc. Sci. Nat. Strasbourg, 5 (1), $14 \mathrm{p}$.

LHONORE L., MAURIN H., GUILBOT R., KEITH P., 1993. Inventaire et cartographie des invertébrés comme contribution à la gestion des milieux naturels français ; actes du séminaire tenu au Mans les 6 et 7 novembre 1992. Collection Patrimoines Naturels, volume 13, Paris, Secrétariat Faune-Flore / MNHN, 214 p.

MACHINO Y., 1995. Austropotamobius torrentium (Schrank, 1803) existe bel et bien en France. L'astaciculteur de France, 45, 17-19.

PRYGIEL J., COSTE M., 1996. Les diatomées et les indices diatomiques dans les réseaux de mesure de la qualité des cours d'eau français: historique et avenir. Bull. Fr. Pêche Piscic., 341-342, 65-79.

TARDIEU V., 1997. 400 naturalistes américains déclarent la guerre aux espèces exotiques. Le monde, 4 avril 1997, p. 24.

TUFFERY G., VERNEAUX J., 1967. Une méthode zoologique pratique de détermination de la qualité biologique des eaux courantes. Indications biotiques. Ann. Sci. Univ. Besançon, Biol. Anim., 3, 79-90.

VERNEAUX J., 1973. Cours d'eau de Franche-Comté. Recherches écologiques sur le réseau hydrographique du Doubs. Mém. Thèse Doct. Etat, Univ. Besançon, 260 p.

VERNEAUX J., 1976. Biotypologie de l'écosystème « eau courante ". La structure biotypologique. C. R. Hebd. Séances Acad. Sci. (D), Paris, 284, 1663-1666.

VIGNEUX D., 1980. Enquête sur les écrevisses en France. Dépouillement et compte rendu. Rapport Conseil Supérieur de la Pêche, $156 \mathrm{p}$.

VIGNEUX E., KEITH P., NOEL P., 1993. Atlas préliminaire des crustacés décapodes d'eau douce de France. Coll. Patrimoines Naturels, Vol. 14, SFF, BIMM - MNHN, CSP, Min. Env., Paris, $55 \mathrm{p}$. 